ORIGINAL ARTICLE

\title{
Health Workers' Awareness and Knowledge of Current Recommendation of Intermittent Preventive Treatment in Pregnancy in South-Western Nigeria
}

\author{
Oluwasomidoyin Olukemi Bello ${ }^{1 *}$, Olaolu Oni ${ }^{2}$
}

\footnotetext{
OPEN ACCESS

Citation: Oluwasomidoyin Olukemi Bello, Olaolu Oni. Health Workers' Awareness and Knowledge of Current Recommendation of Intermittent Preventive Treatment in Pregnancy in South-Western Nigeria. Ethiop J Health Sci.2020;30(1):125. doi:http:// dx.doi.org/ 10.4314/ejhs.v30i1.16

Received: March 17, 2019

Accepted: August 31, 2019

Published: January 1, 2020

Copyright: (C) 2020 Oluwasomidoyin

O.B., et al. This is an open access article distributed under the terms of the Creative Commons Attribution License, which permits unrestricted use, distribution, and reproduction in any medium, provided the original author and source are credited.

Funding: Nil

Competing Interests: The authors declare that this manuscript was approved by all authors in its form and that no competing interest exists.

Affiliation and Correspondence:

${ }^{1}$ Urogynaecology Unit, Department of

Obstetrics and Gynaecology,

University of Ibadan/University

College Hospital, Ibadan, Nigeria

${ }^{2}$ Fetomaternal Medicine, Department of

Obstetrics and Gynaecology,

University College Hospital, Ibadan,

Nigeria

*Email: bellodoyin@yahoo.com
}

ABSTRACT

BACKGROUND: Malaria in pregnancy is of public health significance because of its associated maternal and fetal complications. This study aimed to assess health workers' awareness and knowledge of the current World Health Organisation (WHO) recommendation of intermittent preventive treatment in pregnancy with Sulfadoxine-Pyrimethamine (IPTp$S P)$.

METHODS: A cross-sectional study among 148 health workers who offer obstetrics care in selected health facilities in Ibadan, Nigeria using a self-administered questionnaire to evaluate their awareness and knowledge of the current WHO IPTp-SP. Information on their socio-demographic and professional characteristics, awareness, knowledge and practice of the current IPTp recommendation were obtained. Data analysis involved descriptive and bivariate analyses using SPSS version 20.0 with level of significance set at $p<0.05$.

RESULTS: The majority, 85(57.4\%), of the health workers had been providing obstetrics care for less than 5 years with most of them, 114(77.0\%), practicing at tertiary health facility. More than half, 92(62.2\%), of them were aware of the current WHO IPTp-SP recommendation while about two-fifth (39.1\%) had its correct knowledge. Of the health workers who were knowledgeable of the current IPTp-SP recommendation almost three-quarter, 29(72.2\%) of them prescribed it. The health workers' professional cadre $(p<0.001)$ and duration of providing obstetrics care $(p=0.012)$ were significantly associated with their awareness and correct knowledge of the current IPTp-SP recommendation.

CONCLUSION: Most of the health workers are aware but not knowledgeable of the correct administration of the current IPTp$S P$ recommendation. Likewise, many of them do not prescribe it. This calls for regular training and update of health workers and institutional protocol so as to effectively reduce the prevalence of malaria in pregnancy and its complications.

KEYWORDS: Health worker, IPTp, awareness, knowledge 


\section{INTRODUCTION}

The World Health Organisation (WHO), in 2017, reported an estimate of 219 million cases of malaria worldwide, compared with 217 million cases in 2016 with majority (92\%) of the cases occurring in WHO African Region (1). Of the fifteen countries in sub-Saharan Africa with almost $80 \%$ of the global malaria burden, 5 countries accounted for nearly half of all the malaria cases: Nigeria with $25 \%$ tops the list followed by Democratic Republic of the Congo (11\%), Mozambique (5\%), India (4\%) and Uganda (4\%). The 10 highest burden countries in Africa reported increase in cases of malaria in 2017 compared with 2016. Of these, Nigeria, Madagascar and Democratic Republic of the Congo had the highest estimated increases, all greater than half a million cases (1).

In Nigeria, $97 \%$ of the populations are at risk with pregnant women having 4 times higher increased risk due to changes in their hormone levels with reduction in immunity to malaria and the physiological changes of increased blood flow to the skin which promote attractiveness to mosquitoes $(2,3)$. The relatively high prevalence of $19.7 \%$ to $72.0 \%$ malaria in pregnancy (MiP) is attributed to inadequate and/or ineffective preventive measures, poor knowledge of risks associated with self-medication, negative cultural beliefs, and co-infection of intestinal parasites $(2,4-$ 6). The national uptake of intermittent preventive treatment with sulfadoxine-pyrimethamine (IPTpSP) which is the periodic administration of curative dose of an antimalarial drug-SP to pregnant women as a preventive measure is low. Although this was scaled up from 5 to $15 \%$ from 2008 to 2013 (7-8), the improvement is far below the $80 \%$ national target (8) and the rate of uptake varying from $2 \%$ to more than $60 \%$ with significant regional and socioeconomic disparities in its utilisation rates (9).

MiP is associated with a high rate of maternal and perinatal morbidity and mortality including maternal and fetal anemia, stillbirth, premature delivery and low birth weight $(10,11)$. IPTp-SP at scheduled antenatal visits after the first trimester is an integral part of the WHO three-branched method of preventing and treating malaria during pregnancy. This also includes the use of Insecticide-Treated Nets (ITNs), and a rapid and effective case management. IPTp-SP is beneficial in improving pregnancy outcome in malaria endemic regions like Nigeria (12-14).

Prior to 2012, IPTp-SP guideline involved administration of at least two doses of SP to pregnant women spaced in time at four weeks interval and not given at the later stages of pregnancy because of the concerns of side effect (10). This dosage guideline was updated in October 2012 by the WHO that at each scheduled antenatal clinic (ANC) contact from the second trimester (13th week) to the delivery period, IPTp-SP should be administered monthly at least four weeks apart with a total of 6 doses without safety concerns $(1,14,15,16)$. This policy has been adopted since 2014 (2) but before its adoption, the main strategies for the prevention of MiP in Nigeria were by chemoprophylaxis using two doses of sulphadoxine-pyrimethamine (SP) and use of longlasting ITNs (17). Compliance to uptake of IPTpSP among pregnant women is influenced by several factors. These factors include providerclient relationship, perceived risk-benefits of the drug, previous drug experiences, drug shortages, lack of portable water where directly observed therapy (DOT) is practiced, and drugs not being offered by the health workers (18-20). Additionally, studies have shown that health workers prescribe un-recommended anti-malaria drugs or give wrong dose of SP due to poor knowledge on best practices as a result of confusion on the timing of IPTp doses or unclear policy $(17,21)$. Challenges in health institution on job supervision by stakeholders to ensure compliance with recommended $\mathrm{MiP}$ guidelines and low confidence on the safety of repeated dose of SP also impedes compliance with IPTp-SP (22). It is therefore against this finding that this present study aimed at assessing the awareness and knowledge of the current WHO recommendation of IPTp-SP among health workers in a South-western state in Nigeria four years after it was adopted.

\section{MATERIALS AND METHODS}

This was a 4-month cross-sectional study conducted from 1st February to 31st May 2018 among 148 consenting health workers providing

DOI: http://dx.doi.org/10.4314/ejhs.v30i1.16 
daily obstetrics care during antenatal period in Adeoyo Maternity Hospital (AMH) and University College Hospital (UCH), Ibadan, Oyo state, South western Nigeria. Ibadan is the largest city in West Africa by geographical area and the 3rd largest city in Nigeria with a population of over 3.5 million. Prevalence of malaria in pregnancy in this region is about 4.8-7.7\% between 2011 and 2019 (23).

The Ibadan North local government area (LGA) was purposively selected from the 11 LGAs in Ibadan because it is the only LGA with a tertiary health facility and AMH was randomly selected from the 5 secondary health facilities in the LGA. $\mathrm{UCH}$, the premier teaching hospital in Nigeria, has an average of 8,300 and 1,700 pregnant women attending antenatal and booking clinic yearly while 13,000 and 4,200 attend AMH respectively.

The sample size of 116 was computed with WinPepi version 11.65 using confidence level of $95 \%$ and assuming a minimum of $50 \%$ of health workers would be knowledgeable about the current WHO IPTp-SP with the total population of eligible health workers taken into consideration. All consenting health workers providing antenatal care services in the antenatal clinics and wards of the two hospitals during the study period were interviewed using a self-administered semistructured paper-based questionnaire designed by the investigators and pre-tested to assess for clarity and understanding of the questions and validation prior to its administration.

Information on their socio-demographics and professional characteristics, awareness and knowledge of the current WHO IPTp-SP recommendation and practice, and reasons for nonadherence to the recommendation were obtained. Ethical approval was obtained from the Oyo state ethics review committee and a written informed consent was obtained from the respondent before administration of the questionnaire by trained research assistant. Health workers that were neither nurses nor doctors and those not providing antenatal care services were excluded from the study.
In this study, awareness of the current WHO IPTp-SP was defined as "respondent having heard of the use of monthly IPTp-SP from second trimester to the delivery period adopted in Nigeria in 2014" while full knowledge of all the components of the current WHO IPTp-SP which includes "ability to appropriately identify the gestational age (from the beginning of the 13th week or second trimester) to commence the IPTp$\mathrm{SP}$, the dose $(500 \mathrm{mg}$ Sulphadoxine and $25 \mathrm{mg}$ Pyrimethamine per tablet with three tablets given as one full dose), the frequency (monthly - at least four weeks apart), and the use of IPTp-SP not restricted till delivery period (when to discontinue the use of IPTp-SP in late pregnancy)"was categorized as correct knowledge. The practice of the current WHO IPTp-SP recommendation by the health workers was assessed by self-report of its recommendation/prescription and the actions taken (information given to the pregnant women and its documentation in the case note). In addition, the knowledge of the previous WHO recommendation on IPTp-SP dosing, timing and frequency was assessed.

Data was cleaned, entered and analysed using IBM SPSS version 20.0. Continuous variables were expressed as mean and standard deviation and categorical variables as percentage. The Chi-square test was used to identify the factors associated with respondents' awareness and knowledge of current WHO recommendation of IPTp-SP, and the level of statistical significance was set at $\mathrm{p}<0.05$.

\section{RESULTS}

Of the 148 health workers studied, the majority, $114(77.0 \%$, ) were nurses/midwives while $23.0 \%$ were doctors of which $6.1 \%$ were resident doctors undergoing specialist training in obstetrics and gynaecology. A little above half, 85(57.4\%), had been providing obstetrics care during the antenatal period for less than 5 years while the majority, $114(77.0 \%)$, practiced at the tertiary level of health care. On the average, more than half, 84(56.8\%), of the health workers attended to about 16 pregnant women and above weekly (Table 1). 
Table1: Baseline characteristics of respondents

\begin{tabular}{lll}
\hline Variable & Frequency & Percentage \\
\hline Cadre & & \\
Nurses/midwives & 114 & 77.0 \\
House officer & 25 & 16.9 \\
Obstetrics and Gynaecology Resident doctors & 9 & 6.1 \\
Duration of providing obstetrics care (years) & & \\
$<5$ & 85 & 57.4 \\
$5-9$ & 30 & 20.3 \\
$10+$ & 33 & 22.3 \\
Level of health care facility & & \\
Secondary & 34 & 23.0 \\
Tertiary & 114 & 77.0 \\
Number of pregnant women attended to weekly & & \\
$\leq 5$ & 6 & 4.1 \\
$6-10$ & 25 & 16.9 \\
$11-15$ & 26 & 17.6 \\
$16+$ & 84 & 56.8 \\
No response & 7 & 4.7 \\
\hline
\end{tabular}

Almost all, 140(94.6\%), the health workers had knowledge of the previous WHO recommendation of 2 doses of IPT with SP for MiP at four weeks interval, and $123(83.1 \%)$ knew the correct time for the commencement of its administration while only
$46(31.1 \%)$ had knowledge of required additional dose of IPTp-SP in some medical conditions like sickle cell disease and human immunodeficiency virus positive women (Table 2).

Table 2: Knowledge of Sulphadoxine-Pyrimethamine (SP) for IPT of Malaria in Pregnancy (previous recommendation of 2 doses in pregnancy)

\begin{tabular}{lll}
\hline Variable & Frequency & Percentage \\
\hline Knowledge of IPT of malaria in pregnancy & 140 & 94.6 \\
Yes & 8 & 5.4 \\
No & & \\
Knowledge of onset of administration time for IPTp & 123 & 83.1 \\
Yes (second trimester 13-26 weeks) & 14 & 9.5 \\
No (others) & 11 & 7.4 \\
No response & & \\
Knowledge of conditions for additional IPTp treatment & 46 & 31.1 \\
Yes & 91 & 61.2 \\
No & 11 & 7.4 \\
No response & & \\
\hline
\end{tabular}

Regarding the current WHO recommendation of IPTp-SP, about two-third (62.2\%) of the health workers were aware of it while only $36(39.1 \%)$ of them had the correct knowledge of its administration. The majority $(72.2 \%)$ of the health workers who had correct knowledge of the current WHO IPTp-SP recommendation prescribed it. However, of the general study population, the majority, 133(89.9\%), endorsed adoption of the current WHO recommendation (Table 3). 
Table 3: Awareness and knowledge of current WHO IPTp-SP recommendation

\begin{tabular}{lll}
\hline Variable & Frequency & Percentage \\
\hline Aware of current WHO IPTp-SP recommendation & & \\
Yes & 92 & 62.2 \\
No & 49 & 33.1 \\
No response & 7 & 4.7 \\
Correct knowledge of current WHO IPTp-SP & & \\
recommendation (n=92) & 36 & 39.1 \\
Yes & 56 & 60.9 \\
No & & \\
Prescribe the current WHO IPTp-SP & 26 & 72.2 \\
Yes & 10 & 27.8 \\
No & & \\
Recommendation for adherence to current new WHO IPTp- & \\
SP (n=148) & 133 & 89.9 \\
Yes. & 12 & 8.1 \\
No & 3 & 2.0 \\
No response & & \\
\hline
\end{tabular}

The health workers who were aware, knowledgeable and prescribed the current WHO IPTp-SP recommendation took some actions during the routine prescription. These actions include informing the women on the reason for IPTp, when to take the next dose, the adverse reactions that could occur and to report back if adverse reaction(s) occurs in addition to documenting the prescribed drug in the case note (Figure 1).

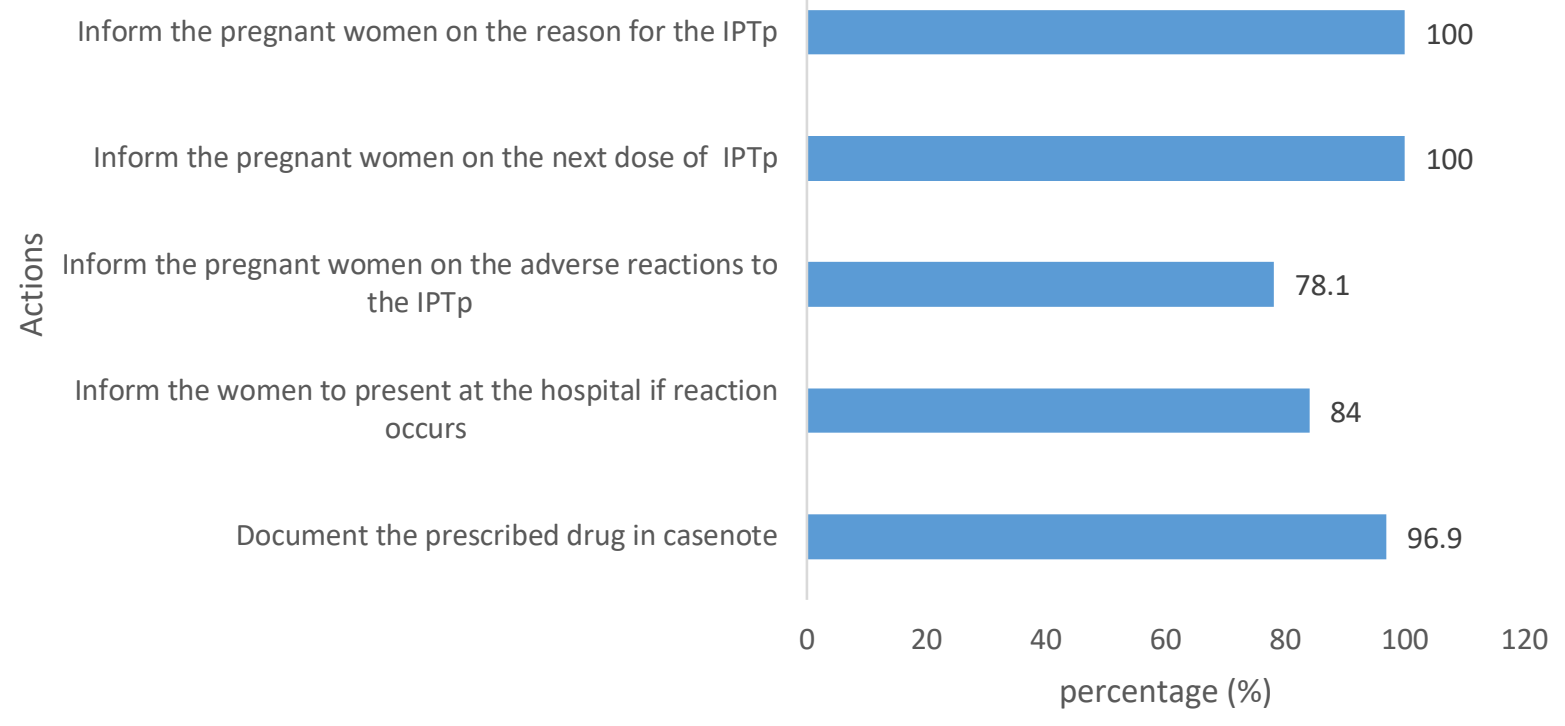

Figure 1: Action taken by health workers during routine IPTp administration

DOI: http://dx.doi.org/10.4314/ejhs.v30i1.16 


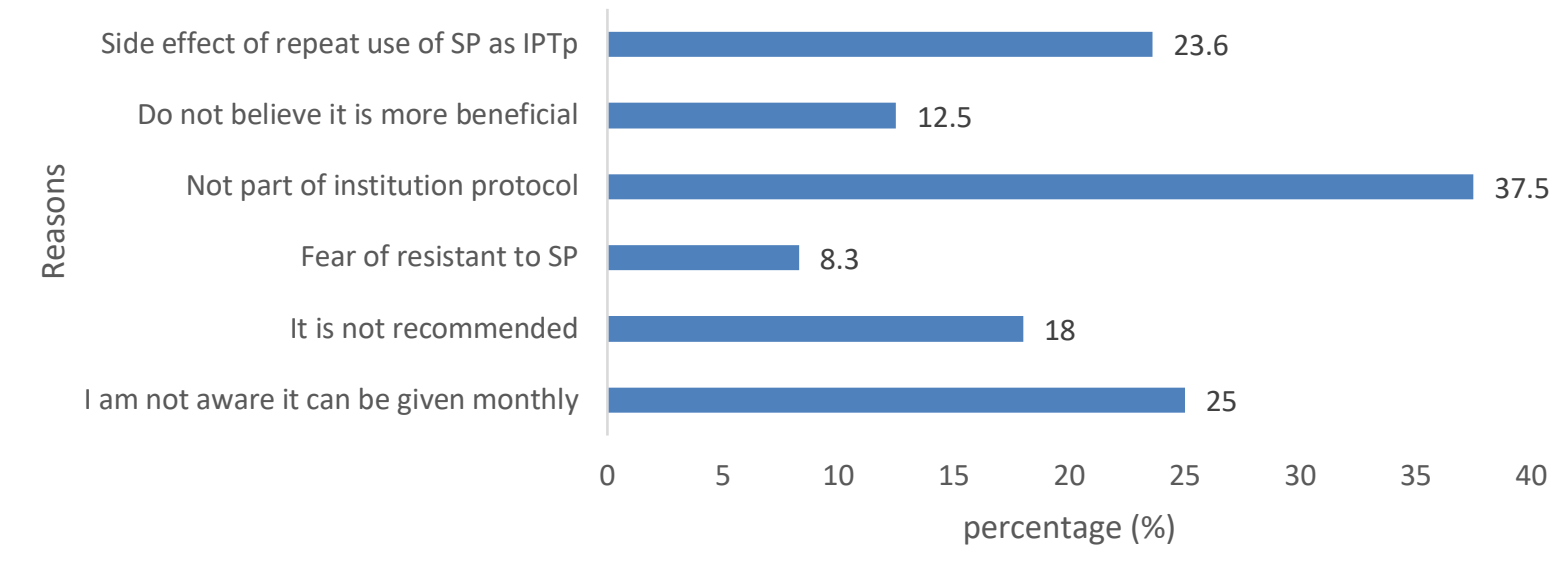

Figure 2: Reasons for non-adherence to current WHO IPTp-SP recommendation (multiple responses among health workers who do not prescribe the current recommendation).

In addition, the health workers who were knowledgeable and prescribed the currently recommended WHO IPTp-SP over the previous recommendation do so because it has fewer side effects $(46.8 \%)$, more effective $(37.6 \%)$, more benefits $(31.4 \%)$ and for the reason that it is recommended by WHO (18.8\%). However, over a third $(37.5 \%)$ of the health workers who were knowledgeable of the current IPTp-SP recommendation did not prescribe it, because it was not part of their hospital protocol while $23.6 \%$ and $8.3 \%$ had the fear that repeated doses is associated with side effects and resistance to SP respectively (Figure 2).

A higher proportion of doctors were aware and had correct knowledge of the current WHO IPTp-SP recommendation $(80.0 \%)$ compared to nurses/midwives $(22.4 \%),(p<0.001)$. Also, the majority of the health workers who had provided antenatal obstetric care for less than five years were aware and had correct knowledge of the current WHO IPTp-SP recommendations compared to those who had practiced for five years and more, $(p=0.012)$. These were statistically significant at $\mathrm{p}<0.001$ and 0.012 respectively. Although not significant, health workers practicing at secondary health facility were aware and had correct knowledge of the current WHO IPTp-SP recommendations compared to those practicing at tertiary health facility $(\mathrm{p}=0.501)$, (Table 4$)$.

\section{DISCUSSION}

The awareness of the current WHO IPTp-SP among the health workers in this study is relatively high while only few of them had the correct knowledge and prescribed it. The finding of low proportion of health workers having the correct knowledge of the current WHO recommendation of IPTp-SP corroborates the report from other studies $(18,22,24,25)$. Most studies in sub-Saharan Africa including Nigeria documented that health workers' inadequate knowledge as key barrier to recommendation of IPTp in both private and public health facilities (22,24-26). Likewise, in a systematic review and meta-analysis of the factors affecting the delivery, access, and use of interventions to prevent MiP, poor knowledge and poor administration of IPTp guidelines by health workers were identified as the most significant barriers to achieving high coverage of IPTp (18). This poor knowledge and practices of IPTp-SP in MiP was similarly reported in Ibadan as far back as 2012 among health workers (21). This trend could depict that the health workers lack enough information, are inadequately trained or unclear of the policy and guideline of the IPTp-SP recommendation. 
Table 4: Association between awareness and correct knowledge of the current WHO SP for IPTp and selected baseline characteristics of the respondents.

\begin{tabular}{|c|c|c|c|c|c|}
\hline \multirow[t]{2}{*}{ Variable } & \multicolumn{2}{|c|}{$\begin{array}{l}\text { Awareness and correct } \\
\text { knowledge }\end{array}$} & \multirow{2}{*}{$\begin{array}{l}\text { Total } \\
\mathbf{N}(\%)\end{array}$} & \multirow[t]{2}{*}{ Chi square } & \multirow[t]{2}{*}{$P$ value } \\
\hline & $\begin{array}{l}\text { Yes } \\
\text { n (\%) }\end{array}$ & $\begin{array}{l}\text { No } \\
\text { n (\%) }\end{array}$ & & & \\
\hline \multicolumn{6}{|l|}{ Cadre } \\
\hline Nurse/midwives & $16(23.9)$ & $51(76.1)$ & $67(100)$ & 24.073 & $<0.001$ \\
\hline Doctor & $20(80.0)$ & $5(20.0)$ & $25(100)$ & & \\
\hline \multicolumn{6}{|c|}{ Level of health care facility } \\
\hline Secondary & $12(44.4)$ & $15(55.6)$ & $27(100)$ & 0.453 & 0.501 \\
\hline Tertiary & $24(36.9)$ & $41(63.1)$ & $65(100)$ & & \\
\hline \multicolumn{6}{|c|}{$\begin{array}{l}\text { Duration of providing } \\
\text { antenatal obstetrics care }\end{array}$} \\
\hline$<5$ & $28(49.1)$ & $29(50.9)$ & $57(100)$ & 6.281 & 0.012 \\
\hline $5+$ & $8(22.9)$ & $27(77.1)$ & $35(100)$ & & \\
\hline \multicolumn{6}{|c|}{$\begin{array}{l}\text { Number of pregnant women } \\
\text { attended to per week }\end{array}$} \\
\hline$\leq 10$ & $8(44.4)$ & $10(55.6)$ & $18(100)$ & 0.769 & 0.681 \\
\hline $11-15$ & $7(46.7)$ & $8(53.3)$ & $15(100)$ & & \\
\hline $16+$ & $21(36.2)$ & $37(63.8)$ & $58(100)$ & & \\
\hline
\end{tabular}

Almost three quarters $(62.2 \%)$ of the respondents were aware of the current IPTp-SP recommendation, but only a few of them recommend in pregnancy. This could probably be attributed to their incorrect knowledge, inability to recall the specifics of the recommendation, different hospital protocol or use of trade names and tablet dosing rather than generic names and strengths of drugs (27). Also, confusion among health workers on the timing and dosing of IPTp with SP administration has been identified as the main barrier slowing the efforts to scale-up IPTpSP in Africa (28). These findings underscore the need to create more awareness and improve specific knowledge on IPTp-SP among frontline health workers that provides antenatal care through training and provision of the current WHO IPTp policy and simplified guideline to improve their practice of delivering IPTp.

As shown in this study, the main actions by the health workers who are aware, knowledgeable and prescribed the current recommended IPTp-SP during routine administration ranged from informing the pregnant women on the reason for the IPTp, when to take the next dose and documentation of the prescribed drug in the case note. This implies that these health workers are knowledgeable of the expected practices during routine IPTp administration (29).
Furthermore, the main reason given by the health workers who were knowledgeable but were nonadherence to prescribing the current recommendation was that the IPTp-SP recommendation was not in their institutional protocol. Other reasons were side effects of repeated use of IPTp-SP, not believing it is more beneficial than the previous recommendation of 2 doses and the fear of resistant to SP. Some of these reasons were like those reported by other researchers in malaria endemic regions regarding the earlier recommendation guideline $(30,31)$. This can be narrowed down to their inadequate knowledge of the details of the current guideline. Therefore, considering these reasons, there is a need for the ministry of health to assess the use of the current WHO recommendation of IPTp in health facilities, and if need be, to disseminate this updated recommendation widely so as to improve its correct prescription and thus its effectiveness in preventing MiP with its resultant maternal and fetal complications. Furthermore, the use of mobile health in complementing classroom training on IPTp which has been shown to significantly improve health workers' knowledge and performance in adhering to guidelines and scaling up of IPTp can be adopted (32).

Additionally, health workers with the knowledge of the current IPTp recommendation who also prescribe it do so because it is recommended by

DOI: http://dx.doi.org/10.4314/ejhs.v30i1.16 
WHO, more beneficial and more effective. This buttress reports from other studies among health workers with similar explanations given as reasons for their compliance in prescribing the recommended drugs for malaria prophylactics at the required dose and time $(33,34)$. Knowledge accrued from trainings or other sources might also contribute a great deal. It is recommended that health workers especially those providing antenatal obstetric care be trained on the current recommendation of IPTp-SP in order to effectively prescribe it. This could be corroborated with Arulogun et al's report in which health workers' main source of IPTp knowledge was through training (21).

Factors significantly associated with health workers' awareness and correct knowledge of the current WHO IPTp-SP recommendation were professional cadre and duration of practice of antenatal obstetric care. The doctors in our study were more aware and had correct knowledge of the current recommendation than the nurses/midwives. This substantiates $\mathrm{Ng}$ 'etich et al report from Kenya on providers' knowledge of the guidelines for IPT for MiP with most clinical officers being knowledgeable about current WHO IPTp -SP compared with the other professional cadres (35). However, it contradicts the report of the survey conducted by Maheu-Giroux and Castro on factors affecting providers' delivery of IPTp, in which clinicians were least likely to deliver IPTp to their clients (36). This might also be because our study was conducted in a secondary and tertiary institution which are referral hospitals where high risk pregnancies are managed. Accordingly, doctors/clinicians attend to women with high risk pregnancy at clinic visit/contact, so they are at the forefront of prescribing IPTp compared to primary health facilities where nurse/midwives, community health extension workers and community health officers attend to low risk pregnant women (37).

Our study showed that health workers providing antenatal obstetrics care for less than five years were more aware and had correct knowledge of WHO IPTp-SP than their colleagues who had been practicing for more than five years. The probable explanation to this is that these set of health workers having less than five years' experience in obstetrics might have received adequate training about the new recommendation prior to commencement of work in ANC of these health facilities. This finding was not in keeping with previous report on providers' knowledge of the guidelines for IPT for MiP as the providers with less than 5 years of professional experience were less knowledgeable about the current IPTp recommendation in Kenya, though the association was not found to be statistically significant on further analysis (35).

Our study has its own limitations. No variable in the questionnaire assessed if the health workers had recent trainings or workshop and the source of information on the current WHO IPTp-SP among those that were knowledgeable. This could have formed a basis for dissemination of updated information on malaria chemoprophylaxis in pregnancy to the health workers. Also, their attitude and constraints were not assessed as this would have given further information for their non-adherence to the current recommendation. In addition, adherence to DOT, contraindications to IPTp-SP and health workers who prescribe based on only the number of tablets/pills to be taken rather than exact dosage and strength was not explored. Conversely, to the best of our knowledge, this is the first study that assessed the health workers' awareness, knowledge and practice of the current WHO recommendation of IPTp-SP in our environment, and the study provides a sample of health workers in one of the 774 LGAs in Nigeria. Though the study cannot be generalized. It will serve as a basis for further studies in other geopolitical zones and among health workers in private and primary health facilities.

However, it is important that institutional guidelines are reviewed and updated with the use of evidence-based practice to include the current recommendation for IPTp-SP. Periodical training on current recommendation should also be organised to upgrade the knowledge of health professionals attending to pregnant women during scheduled antenatal visits.

In conclusion, the knowledge and correct administration of the current WHO recommendation of IPTp-SP among health workers is inadequate. Though a higher proportion among the few ones with correct knowledge prescribes it according to specification of the WHO guideline recommendation, adherence to current WHO IPTp-SP should be encouraged to reduce the prevalence of malaria in pregnancy. 


\section{REFERENCES}

1. WHO (2018). World malaria report, 2018. Accessed online $20^{\text {th }}$ June, 2019 from, https://apps.who.int/iris/bitstream/handle/10665/27 5867/9789241565653-eng.pdf?ua $=1$. Accessed on 29th June, 2019

2. USAID. President's Malaria Initiative: Nigeria malaria Operational Plan FY. Available from: https://www.pmi.gov/.../malaria-operationalplans/fy17/fy-2017-nigeria malaria-opera. (2017). Accessed on 19th August, 2018

3. Lindsay S, Ansell J, Selman C,Cox V, Hamilton K, Walraven G. Effect of pregnancy on exposure to malaria mosquitoes.Lancet 2000;355:1972.

4. Aliyu MM, Nasir IA, Umar YA, Vanstawa AP, Medugu JT, Emeribe AU, et al. Prevalence, risk factors, and antimalarial resistance patterns of falciparum plasmodiasis among pregnant women in Kaduna metropolis, Nigeria. Tzu Chi Med J 2017;29:98-103.

5. Chinweuba AU, Agbapuonwu NE, Onyiapat JE, Israel CE, Ilo CI, Arinze JC. Determinants of Malaria Prevention and Treatment Seeking Behaviours of Pregnant Undergraduates Resident in University Hostels, South-East Nigeria. Journal of Pregnancy Volume 2017, Article ID 3653874.

6. Akinbo FO, Olowookere TA, Okaka CE, Oriakhi MO. Co-infection of malaria and intestinal parasites among pregnant women in Edo State, Nigeria. J Med Trop 2017;19:43-8.

7. National Population Commission (NPC) (Nigeria) and ORC Macro. Nigeria Demographic Health Survey (NDHS 2008). Calverton MNPC and OM. 2008.

8. Olugbade OT, Ilesanmi OS, Gubio AB, Ajayi IO, Nguku PM, Ajumobi OO. Socio-demographic and regional disparities in utilization of intermittent preventive treatment for malaria in pregnancy Nigeria demographic health survey 2013. The Pan African Medical Journal. 2019;32 (Supp 1):13.

9. Steketee RW, Eisele TP. Is the scale-up of malaria intervention coverage also achieving equity? PLoS ONE 2009, 4.

10. Esu E, Effa E, Udoh E, Oduwole O, Odey F, Chibuzor $\mathrm{M}$ et al. Utilization of intermittent preventive treatment for malaria among pregnant women attending antenatal clinics in health facilities of Cross River State, Nigeria. Research and Reports in Tropical Medicine 2013; 4:29-35.

11. Agboghoroma CO. Current management and prevention of malaria in pregnancy: a review. West Afr J Med 2014; 33:91-99.
12. World Health Organization (WHO). Updated WHO Policy Recommendation: Intermittent Preventive Treatment of malaria in pregnancy using Sulfadoxine-Pyrimethamine (IPTp-SP). Available from http://www.who.int/malaria/iptp_sp_updated_polic y_recommendation_en_102012.pdf. (October 2012). Accessed on 19th August, 2018.

13. Aduloju OP. Effect of intermittent preventive treatment of malaria on the outcome of pregnancy among women attending antenatal clinic of a new Nigerian teaching hospital, Ado-Ekiti. Niger Med J 2013; 54: 170-5.

14. Agomo CO, Oyibo WA, Odukoya-Maije F. Parasitologic Assessment of Two-Dose and Monthly Intermittent Preventive Treatment of Malaria during Pregnancy with SulphadoxinePyrimethamine (IPTP-SP) in Lagos, Nigeria. Malaria Research and Treatment 2011; 2011: 6:e0146908.

15. World Health Organization (WHO).The Global Strategic Plan 2005-2015. Geneva: WHO; Roll Back Malaria. Available from: http://www.rollbackmalaria.org/forumV/docs/gsp_ en.pdf. (2005). Accessed on 19th August, 2018.

16. WHO Antenatal Care Guidelines: Malaria in Pregnancy Frequently Asked Questions (FAQ) 2016. Available from:

https://www.mcsprogram.org/resource/2016-whoantenatal-care-guidelines-malaria-in-pregnancyfrequently-asked-questions-faq/ Accessed on 5th June, 2019

17. Federal Ministry of Health. National guidelines and strategies for malaria prevention and control during pregnancy. Federal Ministry of Health, Abuja, Nigeria. Available from https://aidsfree.usaid.gov/sites/default/files/tx_nige ria_pmtct_2010.pdf. (2005). Accessed on 19th August, 2018.

18. Hill J, Hoyt J, van Eijk A, D'Mello-Guyett L, Kuile F, Steketee R, et al. Factors Affecting the Delivery, Access, and Use of Interventions to Prevent Malaria in Pregnancy in Sub-Saharan Africa: A Systematic Review and Meta-Analysis. PLoS Med; 2013; 10(7): e1001488.

19. Matsui D. Adherence with drug therapy in pregnancy. Obstet Gynecol Int. 2011; 2012: 796590.

20. Odongo CO, Bisaso RK, Byamugisha J, Obua C. Intermittent use of sulphadoxine pyrimethamine for malaria prevention: a cross-sectional study of knowledge and practices among Ugandan women 
attending an urban antenatal clinic. Malar J 2014; 13: 399 .

21. Arulogun OS, Okereke CC. Knowledge and practices of intermittent preventive treatment of malaria in pregnancy among health workers in a southwest local government area of Nigeria. $J$ Med Med Sci. 2012; 3: 415-22.

22. Rassi C, Graham K, Mufubenga P, King R, Meier J, Gudoi SS. Assessing supply-side barriers to uptake of intermittent preventive treatment for malaria in pregnancy: a qualitative study and document and record review in two regions of Uganda. Malar J. 2016;15:341.

23. Isah AY, Amanabo MA, Ekele BA. Prevalence of malaria parasitemia amongst asymptomatic pregnant women attending a Nigerian teaching hospital. Ann Afr Med 2011;10:171-4.

24. Rabiu KA, Davies NO, Nzeribe-Abangwu UO, Adewunmi AA, Akinlusi FM, Akinola OI et al. Malaria prevention and treatment in pregnancy: Survey of current practice among private medical practitioners in Lagos, Nigeria. Trop Doct 2015; 45: 6-11.

25. Thiam S, Kimotho V, Gatonga P. Why are IPTp coverage targets so elusive in sub-Saharan Africa? A systematic review of health system barriers Malar J. 2013, 12:353

26. Diala C, Pennas T, Marin C, Belay K. Perceptions of intermittent preventive treatment of malaria in pregnancy (IPTp) and barriers to adherence in Nasarawa and Cross River States in Nigeria. Malar J. 2013;12 Suppl 1:342.

27. World Health Organization resource. Essential Medicines and Health Products Information Portal, Guide to Good Prescribing - A Practical Manual. (1994). Available at: https://apps.who.int/medicinedocs/en/d/Jwhozip23 e/5.4.html. Accessed on 29th August, 2019.

28. World Health Organization (WHO). WHO policy brief for the implementation of intermittent preventive treatment of malaria in pregnancy using sulfadoxine-pyrimethamine (IPTp-SP). (2014). Available at: https://www.who.int/malaria/publications/atoz/poli cy brief iptp sp policy recommendation/en/ Accessed on 19th August, 2018.

29. Onoka C, Onwujekwe O, Hanson K, Uzochukwu B. Sub-optimal delivery of intermittent preventive treatment for malaria in pregnancy in Nigeria: influence of provider factors. Malar J 2012 11:317.
30. Steketee RW, Nahlen BL, Parise ME, Menendez C The Burden of Malaria in Pregnancy in MalariaEndemic Areas. Breman JG, Egan A, Keusch GT, editors. Northbrook (IL): American Society of Tropical Medicine and Hygiene; 2001

31. Mubyazi G1, Bloch P, Kamugisha M, Kitua A, Ijumba J. Intermittent preventive treatment of malaria during pregnancy: a qualitative study of knowledge, attitudes and practices of district health managers, antenatal care staff and pregnant women in Korogwe District, North-Eastern Tanzania. Malar J. 2005 Jul 20;4:31.

32. Rassi C, Gore-Langton GR, Gidudu Walimbwa B, Strachan CE, King R, Basharat S, et al. Improving health worker performance through text messaging: A mixed-methods evaluation of a pilot intervention designed to increase coverage of intermittent preventive treatment of malaria in pregnancy in West Nile, Uganda. PLoS One. 2018;13(9):e0203554.

33. Akinleye SO, Falade $\mathrm{CO}$ and Ajayi IO. Knowledge and utilization of intermittent preventive treatment for malaria among pregnant women attending antenatal clinics in primary health care centers in rural southwest, Nigeria: a cross-sectional study. BMC Pregnancy Childbirth 2009; 9:28.

34. Amoran OE, Ariba AA, Iyaniwura CA. Determinants of intermittent preventive treatment of malaria during pregnancy (IPTp) utilization in a rural town in Western Nigeria. Reprod Health 2012; 9:12.

35. Ng'etich M, Angela C, Tom O. "Providers' Knowledge of the Guidelines for Intermittent Preventive Treatment for Malaria in Pregnancy: Evidence from Bungoma East District, Kenya." American Journal of Public Health Research 2014; 2: $125-35$

36. Maheu-Giroux M, Castro MC. Factors affecting providers' delivery of intermittent preventive treatment for malaria in pregnancy: a five-country analysis of national service provision assessment surveys. Malar J 2014; 13:440.

37. Ruwei H, Yu L, Zhicheng D, Yuantao Hao, Hailun L, Leiyu S. Types of health care facilities and the quality of primary care: a study of characteristics and experiences of Chinese patients in Guangdong Province, China. BMC Health Serv Res 2016;16: 335. 